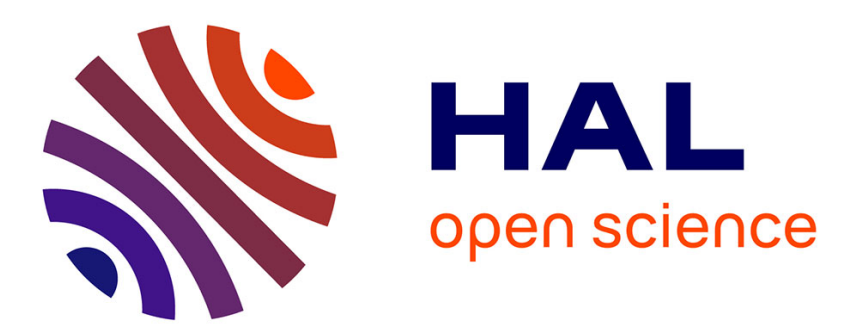

\title{
La question de la réversibilité du démantèlement chez un adulte autiste
}

\author{
Nathalie Barabé, Chantal Lheureux Davidse
}

\section{To cite this version:}

Nathalie Barabé, Chantal Lheureux Davidse. La question de la réversibilité du démantèlement chez un adulte autiste. Cliniques méditerranéennes, 2004, Haïr, ignorer, 2 (70), pp.287 - 306. 10.3917/cm.070.0287 . hal-01513788

\section{HAL Id: hal-01513788 \\ https://hal.science/hal-01513788}

Submitted on 25 Apr 2017

HAL is a multi-disciplinary open access archive for the deposit and dissemination of scientific research documents, whether they are published or not. The documents may come from teaching and research institutions in France or abroad, or from public or private research centers.
L'archive ouverte pluridisciplinaire HAL, est destinée au dépôt et à la diffusion de documents scientifiques de niveau recherche, publiés ou non, émanant des établissements d'enseignement et de recherche français ou étrangers, des laboratoires publics ou privés. 


\section{LA QUESTION DE LA RÉVERSIBILITÉ DU DÉMANTÈLEMENT CHEZ UN ADULTE AUTISTE \\ Nathalie Barabé, Chantal Lheureux-Davidse}

\section{ERES | «Cliniques méditerranéennes »}

2004/2 n 70 | pages 287 à 306

ISSN 0762-7491

ISBN 2749202701

Article disponible en ligne à l'adresse :

http://www.cairn.info/revue-cliniques-mediterraneennes-2004-2-page-287.htm

\section{Pour citer cet article :}

Nathalie Barabé, Chantal Lheureux-Davidse, « La question de la réversibilité du démantèlement chez un adulte autiste », Cliniques méditerranéennes 2004/2 ( $\mathrm{n}^{\circ}$ 70), p. 287-306.

DOI $10.3917 / \mathrm{cm} .070 .0287$

Distribution électronique Cairn.info pour ERES.

(C) ERES. Tous droits réservés pour tous pays.

La reproduction ou représentation de cet article, notamment par photocopie, n'est autorisée que dans les limites des conditions générales d'utilisation du site ou, le cas échéant, des conditions générales de la licence souscrite par votre établissement. Toute autre reproduction ou représentation, en tout ou partie, sous quelque forme et de quelque manière que ce soit, est interdite sauf accord préalable et écrit de l'éditeur, en dehors des cas prévus par la législation en vigueur en France. Il est précisé que son stockage dans une base de données est également interdit. 


\section{Nathalie Barabé Chantal Lheureux-Davidse}

\section{La question de la réversibilité du démantèlement chez un adulte autiste}

Les écrits psychanalytiques sur la clinique de l'autisme infantile font état d'un investissement problématique de la zone buccale et de nombreux troubles sensoriels. Suite à la perte de la consensualité entre les différentes modalités sensorielles, due au démantèlement, la mise en place d'un autoérotisme oral n'a pu se produire.

Le démantèlement défini par $\mathrm{D}$. Meltzer a été décrit à partir de la prise en charge d'enfants autistes. Il est présenté comme réversible. Mais qu'en estil des conséquences du démantèlement à long terme ? Peut-on continuer à parler de réversibilité du démantèlement chez un adulte autiste, utilisant ce mécanisme depuis de nombreuses années?

À partir du cas de Luc, adulte autiste, nous témoignerons de nos observations sur ces points. Nous présenterons d'une part les données cliniques mais également l'aménagement du cadre de travail qui a été nécessaire pour une prise en charge psychothérapique. La mise en place du transfert et sa spécificité seront exposées.

Nous jalonnerons ce texte de nouvelles hypothèses théorico-cliniques sur l'éveil de la zone buccale et sur le rôle des sensations de pression en lien avec des vécus intra-utérins. Ce cas nous paraît d'autant plus riche d'un point de vue clinique qu'il existe peu d'écrits sur des adultes autistes. De plus il permet d'aborder la question de la pertinence d'un travail thérapeutique dans des cas où les troubles sensoriels sont massifs.

Nathalie Barabé, psychologue clinicienne, psychothérapeute, enseignante, Centre de Recherches Cliniques Psychanalytique, Sociale et Culturelle, Paris XIII.

Chantal Lheureux-Davidse, psychologue clinicienne, psychanalyste, enseignante Université Paris VII. 


\section{L'HOMME « TOUT EN BOUCHE »}

Luc est un autiste mutique d'une quarantaine d'années qui semble mettre tout en œuvre pour qu'on ne le regarde pas. De nombreuses stéréotypies déforment son faciès et le rendent très impressionnant. Il est le seul adulte polyhandicapé que je n'ai pas pu regarder lors de ma première visite dans le service ${ }^{1}$. Sa stéréotypie la plus impressionnante consiste à utiliser sa langue comme un aliment qu'il mangerait. Il la fait tourner dans sa bouche, les joues gonflées, la sortant et la rentrant, dans une mimique totalement disgracieuse qui déforme son visage. De plus, il présente un strabisme totalement réversible selon les moments, qui traduit la désorganisation de son regard. L'ensemble suscite un mouvement de recul. Ne possédant que deux dents, il arrive également à remonter le bas de son visage si près de son palais que sa bouche semble alors occuper tout l'espace de son visage.

C'est un homme aux jambes maigres et de taille plutôt petite. Il est souvent assis en tailleur avec une très grande souplesse des articulations de ses membres inférieurs. La plupart du temps, il s'enlace la cage thoracique de son bras droit, dans une posture qui rappelle celle d'un bras coincé par une camisole. Son mode de déplacement est également particulier puisqu'il avance en oscillant de droite à gauche, tel un jeune enfant commençant à marcher ou tel un culbuto, tant l'oscillation entre les pôles est marquée. Sa silhouette maigre, nantie d'une grande couche enveloppant tout son bassin, donne l'impression que la fluidité de ses mouvements est entravée. Cependant, même sans couche, il avance ainsi, dodelinant de droite à gauche.

Il a l'habitude de passer ses journées dans un coin du foyer qu'il s'est approprié. Il a peu d'initiatives durant ses journées, en dehors du fait de tenter de chaparder de la nourriture, soit en déambulant jusqu'à la porte de la cuisine soit en se servant dans l'assiette de ses voisins de table. Ses comportements oraux sont prévalents.

Luc mâchonne sa langue, ou toute sorte d'objets, reproduisant ainsi les sensations tactiles de nourrissage. Il utilise également à cette fin ses gélules de médicaments, petits bouts ronds qu'il fait aller et venir dans sa bouche pendant parfois une heure. Lorsqu'il lui restait des dents, il poussait celles-ci avec sa langue, jusqu'à faire vaciller l'une d'elle sur sa gencive, ce qui lui permettait d'obtenir d'intenses sensations. Il peut également porter des brindilles à sa bouche, lors des pique-niques qu'il apprécie beaucoup. Un jour, en

1. Luc a été suivi en institution par Nathalie Barabé. Ainsi certains passages du texte sont écrits à la première personne du singulier. 
séance, il a décroché du mur une épingle très pointue, l'a mise en bouche, l'a gardée sur sa langue, pour obtenir ainsi des sensations de piqûre.

\section{UN ABORD ORAL DE L'ESPACE}

Luc utilise également sa langue comme une main, pour toucher. Souvent, après avoir plaqué son buste contre le dessus d'une table, il descend de plus en plus sa tête, jusqu'à prendre contact avec cette surface par le bout de sa langue et reste ainsi, plusieurs minutes. Il peut également déposer un petit filet de bave, de temps en temps, à un endroit très précis. Si la langue est un appendice buccal aux qualités tactiles très développées qui exerce diverses fonctions, Luc, en portant tout à la bouche tel un nourrisson, semble étayer ainsi sensoriellement sa conscience des volumes et surfaces. En effet, une des fonctions de la langue est de construire sensoriellement l'orientation spatiale et la conscience des directions.

En séance de musicothérapie, Luc explore avec sa langue les tables et certains petits objets nouveaux qu'il a repérés à distance. Lorsqu'il cherche à entrer en contact avec son environnement, il le fait de façon tactile en posant sa langue sur un objet qu'il a aperçu préalablement. Il le tâte ensuite de sa main droite en le pressant un peu, comme s'il en évaluait la texture. Par contre, il ne s'attarde pas dans la contemplation visuelle : dès que ces contacts tactiles avec sa langue et ses mains sont pris, il s'éloigne des objets sans même les regarder. C'est comme si ces deux minutes d'exploration par le toucher lui suffisaient.

Ceci rappelle le nourrisson qui apprécie l'élasticité, la densité, la profondeur et le confort du sein maternel dans une exploration manuelle et corporelle structurante qui concourt à son accès à la tri dimensionnalité.

À d'autres moments, Luc recherche avec sa langue des sensations tactiles de picotements, par exemple avec des miettes de pain ou avec une épingle. Il lui arrive également de se gratter la langue avec ses ongles. Ces sensations habituellement douloureuses lui permettent-elles de réanimer une zone buccale dont il aurait perdu la perception?

\section{LES SENSATIONS DÉLICIEUSES CONSTITUANTES D'UNE ZONE BUCCALE}

Ainsi, à la piscine, sous la douche, il sort avec délice sa langue, pour y sentir ruisseler des gouttelettes d'eau qui lui procurent des sensations tactiles voluptueuses. Il reste alors longtemps la langue sortie, pointée en avant, le visage immobile. Il obtient également ces sensations en se grattant la langue, geste qu'il fait quotidiennement, tout en plissant les yeux et en émettant un grognement de satisfaction. La vie de cet homme semble guidée par ces sen- 
sations tactiles de picotements, de chatouilles, de brûlures ou de piqûres tant dans la zone de la bouche que sur tout son corps.

Au début de nos rencontres, je repérais chez lui deux formes de tonicité buccale. Soit sa bouche était sans tonus avec la langue pendante laissant écouler passivement de la salive ; soit sa bouche était fermée quand il " mâchonnait » dans le vide, traduisant une sphinctérisation possible de cet orifice. Au fur et à mesure des années de travail avec lui, sa zone buccale se tonifiera de plus en plus.

Au début de nos rencontres, les mâchonnements m'ont paru comme des tentatives de recréer $\mathrm{du}$ « bon » et de l'incorporer. Ces tentatives sont à renouveler constamment, faute d'une intériorisation possible. Cette difficulté à construire et maintenir la conscience des orifices, dont les sensations ne cessent de s'effacer lors des démantèlements sensoriels, est typique des organisations autistiques. Faute d'une permanence d'intériorisation qui bloque l'accès à la tridimensionnalité, certains autistes vivent leur corps comme un ensemble de surfaces, de muqueuses ou de substances liquides ${ }^{2}$.

Chez Luc, on constate que la langue a une triple fonction : celle de «bouchon ", celle de réanimer les sensations de l'orifice buccal et celle d'étayer une prise de conscience de l'espace et des volumes.

\section{UN MONDE DE SURFACE}

Luc investit l'espace de façon uni ou bi-dimensionnelle, selon la théorie de D. Meltzer ${ }^{3}$. Il prend essentiellement en considération les objets ou parties d'objet qui vont lui procurer des sensations de surface et semble annuler les autres dans un mouvement hallucinatoire négatif. Il passe beaucoup de temps à plaquer son buste sur des tables ou sur les instruments de musique, en séance, dans un collage adhésif caractéristique des états autistiques. Cette adhésivité lui permet de faire face à des angoisses primitives d'écoulement ou de vidage en recherchant les limites de son corps par des contacts de surface qui font bord tel un coffrage. Winnicott décrit ces angoisses primitives dans son article sur la crainte de l'effondrement ${ }^{4}$. Le mécanisme défensif consistant à adhérer à une surface dure est également développé par F. Tus-

2. F. Tustin, The protective shell in children and adults, London, Karnac books, 1990 ; trad. franc. : A.L. Hacker, Autisme et protection, Paris, Le Seuil, 1992, p. 62-75.

3. D. Meltzer, « La dimensionalité comme paramètre du fonctionnement mental : sa relation à l'organisation narcissique », chapitre IX, dans Explorations in Autism, Roland Harris Trust, Clunie Press, 1975 ; trad. franç. Explorations dans le monde de l'autisme, Paris, Payot, 1980, p. 232-248. 4. D.W. Winnicott, «La crainte de l'effondrement », dans Nouvelle revue de psychanalyse, 1975, 11, p. $35-44$. 
tin 5 , à travers son concept de recours à des objets autistiques pour rechercher un contact dur et rassurant.

En effet, comme l'a montré E. Bick 6, dans le développement normal du bébé, lors d'angoisses de chute, celui-ci a le réflexe de rigidifier son corps en contractant l'ensemble de ses muscles. Ceci lui procure une sensation de maintien lui rappelant le serrage rassurant de la paroi utérine associé à la portance du liquide amniotique. Pour lutter contre ses angoisses de chute, le bébé est également rassuré par un contact adhésif suffisamment ferme lors du corps à corps avec autrui.

Dans l'autisme, pour lutter contre des angoisses de chute ou d'écoulement, on retrouve l'auto-maintien, la rigidification musculaire tout comme la recherche de prise de contact adhésive avec des surfaces dures.

Dans la salle de musicothérapie, Luc se colle parfois entre la porte et le mur pour colmater l'espace vide de l'interstice. Un jour, il a plaqué son buste contre une personne référente qu'il appréciait, dans un abord d'autrui en tant que surface. De même, il s'assoit de façon très particulière, en se mettant toujours sur le bord des chaises ou des escaliers, sans pouvoir encore investir le contenant ni le fond des objets, qui sont réduits à des bords et à des surfaces de contact.

Il recherche également des sensations d'élasticité des textures associées à l'éprouvé des bords, quand il s'adosse contre une grosse bouée en en testant légèrement le rebond avec tout son corps. Il semble ainsi retrouver des sensations d'élasticité et de profondeur du contenant utérin.

Chez le bébé, cette recherche d'élasticité se prolonge normalement lorsqu'il explore avec ses joues puis ses mains le confort du sein maternel.

Concernant Luc, il peut apprécier la qualité élastique d'une surface, mais il ne perçoit pas encore la notion de profondeur des objets. Ainsi, lors d'une sortie sur un parcours de santé, Luc ne comprenait pas pourquoi il devait se pencher pour passer sous des rondins. Il cherchait juste à se plaquer contre la surface du premier morceau de bois, sans pouvoir envisager la notion d'ouverture ni de profondeur.

Le monde ne semble exister pour lui que par les sensations procurées par les qualités de surface et de bord, quels qu'en soient les supports.

5. F. Tustin, Autistic Barriers in Neurotic Patients, Londres, Karnac Books ; trad. franc. Le trou noir de la psyché, Paris, Le Seuil, octobre 1989, p. 78.

6. E. Bick, 1968, « The experience of the skin un early object-relations, int. », Journal of psychoanalysis, 49, 484-6 ; trad. franç. G. et M. Haag, " L'expérience de la peau dans les relations d'objet précoces ", dans D. Meltzer et coll. (sous la direction de), Explorations dans le monde de l'autisme, 1975, p. 240-244. 
C'est à partir de cette redéfinition du monde en tant que « multitude de surfaces » indifférenciées que Luc exerce des clivages et des choix préférentiels.

\section{DiFFÉRENCIATIONS}

Luc sélectionne dans ce qui l'entoure, des objets aux qualités tactiles subjectivement douces et plaisantes dans une indifférence quant à l'aspect animé, inanimé, humain ou inerte. Pour le choix de ses vêtements, par exemple, il malaxe le tissu avec ses paumes, avant de le porter contre son visage et, si les sensations lui conviennent, il y enfouit sa joue, dans un petit gémissement de plaisir.

Les choix des habits qu'il accepte de porter se fait selon des critères très précis : certaines textures lui provoquent des sensations de peau insupportables, ce qui l'avait amené à se gratter à sang et à arracher sa peau au point d'être mis sous calmants. Pendant cette période d'un an, il avait perdu énormément de poids. Si l'enveloppe représentée par les vêtements peut-être vécue comme bon contenant, selon le concept de Moi-peau de D. Anzieu 7, elle ne l'était pas pour lui. Il ne ressent ses vêtements que par leurs qualités de surface, parfois persécutrices, avant même de pouvoir les vivre comme bon contenant. En effet, dans un univers bidimensionnel, l'enveloppe représentée par les vêtements se réduit à un contact tactile.

Un jour où il lui est demandé de choisir un canapé dans un magasin, il se lance également dans une exploration tactile des tissus. Il passe plusieurs fois sa paume sur les dossiers et lorsque les sensations de texture lui conviennent, il plaque son buste contre ceux-ci. Globalement, ce sont les qualités de surface qui guident ses choix.

Dans cette vision réduite des objets en tant que surfaces, les orifices deviennent des trous à travers lesquels des objets passent, tombent, sans pouvoir être contenus par une quelconque cavité, à l'image de son corps d'une minceur extrême en dépit de son hyperphagie. C'est comme si physiologiquement, son corps ne retenait rien. Lorsqu'il s'alimente, sa bouche semble réduite à un ensemble de surfaces tactiles dont il cherche à tirer le plus de sensations possibles. 
LA BOUCHE ET L'ORIFICE BUCCAL EN TANT QUE MULTITUDE DE SURFACES TACTILES

Lors de l'alimentation, l'investissement de la zone orale par Luc révèle un démantèlement sensoriel et une impossibilité d'accéder à la tridimensionnalité. L'acte de manger s'en trouve modifié. Ainsi, lors des repas, il porte le maximum d'aliments à sa bouche, quitte à les enfoncer avec la main car il apprécie les grosses bouchées qui dilatent ses muqueuses. Déglutir lui est très difficile. Il « retient » le plus longtemps possible ses aliments, qu'il presse contre ses muqueuses. Lorsqu'on lui demande d'avaler, il ne le fait qu'en serrant très fort son buste avec l'un de ses bras, comme s'il risquait de perdre tout ce qui pouvait lui donner une cohésion interne en avalant. S'agirait-il d'une crainte archaïque de s'auto-avaler en même temps que la nourriture, angoisse que nous avons retrouvée chez des enfants autistes 8 ? D'une part il substitue la pression de son bras sur son buste à celle du bol alimentaire sur ses muqueuses buccales, dans une indifférenciation quant à la localisation de la sensation de pression. $\mathrm{D}^{\prime}$ autre part, en se tenant le buste, il retiendrait son corps d'un risque d'auto-avalement, d'un écoulement infini mortel.

En effet, lors de la déglutition, Luc éprouve un certain nombre de sensations diverses. C'est un moment très fort pour lui. Au moment d'avaler, il recherche une dilatation maximale de la glotte et de la trachée pour intensifier ses sensations. Il accumule à l'extrême les aliments pour obtenir cet effet.

Cette perception très intense de ces zones au moment $d$ 'avaler coexiste avec des angoisses de perte.

De plus, la langue de Luc présente au fond des petits boutons, résistants à un traitement contre le muguet et qui semblent ici renforcer la sensibilité tactile au passage des aliments. Le risque de perte de sensations tactiles sur les parois buccales et les angoisses de disparition liées à la déglutition sont si fortes, que même lors de spasmes de vomissements, il garde précieusement le liquide en bouche quitte à éprouver des sensations d'étouffement. Il faut alors l'encourager à ouvrir la bouche pour laisser partir le liquide. Lorsqu'il mange certains poissons, Luc arrive aussi, par réaction allergique, peut-être, mais également par ses performances corporelles, à excréter de la mousse qui remonte de la trachée jusqu'à la bouche et au nez. Dans quelle mesure la douleur ne serait pas recherchée par Luc afin de conserver plus longtemps une trace de la sensation obtenue ? Nous rejoignons ici le point de vue de

8. Ces angoisses d'auto-avalement chez les enfants autistes ont été travaillées dans le sens d'une tentative d'incorporation d'une scène primitive par la nourriture. C. Lheureux-Davidse, « La relation cannibalique par incorporation pour se faire un corps ", dans L'autisme infantile ou le bruit de la rencontre, Paris, L'Harmattan, 2003. 
P. Fédida ${ }^{9}$ qui parle pour certains patients, de « tentatives de faire exister l'orifice».

Lors de la déglutition trois aspects seraient à différencier : la perte du « colmatage » de l'orifice et de la sensation de fermeture, le risque d'un autoavalement et la perte des sensations de pression sur les récepteurs dermiques. Ce dernier aspect relève plus de la biologie, mais ce sont bien des sensations de pression que Luc met en scène.

\section{LES SENSATIONS DE PRESSION ET LE REGARD CONTENANT}

D'un point de vue neurologique, il n'existe pas de nerf réservé aux sensations tactiles en général, mais différents récepteurs qui sont spécialisés dans des qualités tactiles précises : certains dans les sensations de pression, d'autres dans la perception de la chaleur, etc. Il y a un éprouvé de douleur lorsqu'un circuit de transmission de l'information perceptive n'est plus assez stimulé. Par exemple, s'il n'est plus sollicité ou excité. C'est un principe général du fonctionnement neuronal. Ainsi, lorsqu'un membre a été amputé, les sensations douloureuses liées à cette zone sont transmises au cerveau. L'absence de stimuli perçus provoque la douleur. Ainsi, on peut imaginer des douleurs dues aux récepteurs tactiles de pression insuffisamment stimulés, qui ne seraient pas visibles ou perçues par l'environnement, étant donné le bon fonctionnement des autres récepteurs tactiles au même endroit. Cette hypothèse nous paraît importante quant à la compréhension de la recherche de sensations de pression par Luc et par d'autres autistes.

De plus, la peau est constituée de trois couches différentes : l'épiderme, le derme et l'hypoderme. Certains récepteurs accueillent les sensations douces les plus légères à la surface de la peau, alors que d'autres réagissent aux pressions plus profondes. Dans l'autisme, il semble que les sensations de pression souvent recherchées, aient un effet contenant pour les sensations plus douces, légères, difficilement supportées (les caresses, etc.) et qu'elles jouent ainsi un rôle de pare-excitation. Ce sont en effet les sensations douces qui procurent des émotions trop intenses pour les autistes qui n'arrivent pas à les contenir. La personne autiste serait en manque de sensations de pression pour obtenir l'apaisement.

$C^{\prime}$ est de cet apaisement par la pression dont parle Temple Grandin, dans son livre Ma vie d'autiste ${ }^{10}$, lorsqu'elle raconte combien sa tension, sa nervosité et sa difficulté à être en contact avec les autres se sont apaisées à partir

9. P. Fédida, «L'anatomie dans la psychanalyse », dans Nouvelle revue de psychanalyse, $1971, \mathrm{n}^{\circ}$ 3, p. $109-126$.

10. T. Grandin, Ma vie d'autiste, Paris, Odile Jacob, 1994. 
du moment où elle a fabriqué une « machine à serrer » qu'elle utilisait pour réguler ses états de tension. Elle pouvait se serrer le thorax avec suffisamment d'intensité pour calmer l'excitation insupportable d'une stimulation trop douce qu'elle avait subie sur sa peau et qui n'étaient pas régulée par des sensations de pression suffisamment fortes pour contenir les émotions que la douceur lui procurait.

Luc réagit également comme s'il avait à certains moments une peau «à vif ». Cela fait écho au "traumatisme » psycho-corporel que représente la naissance. En effet, intra-utero, le fœetus est soumis à une excitation relativement constante, par la pression du liquide amniotique sur ses récepteurs dermiques. Ce qui a un effet contenant. Il reçoit une pression aquatique enveloppante relativement constante. La naissance met tout le corps du nouveau-né à vif. Le bébé est alors soumis à des sensations de peau en surface. Il est privé des sensations de pression qui le contenaient. Cette référence à l'état fœtal n'est pas anodine. Luc passe en effet beaucoup de temps à s'humidifier la peau du visage avec sa propre salive et à manifester des comportements évoquant des recherches de sensations fœtales qu'il tente de recréer. On peut supposer que lors de l'état fotal les sensations ne seraient pas encore différenciées, le mouillé serait alors confondu avec la pression contenante. Ainsi, en s'humidifiant la peau, Luc rechercherait par là-même, des sensations de pression.

De même, le nouveau-né découvre des sensations nouvelles de pression sur ses muqueuses buccales lors de la tétée, qu'il associe alors au regard et aux bras contenants de sa mère. Le regard de l'autre pourrait être ainsi vécu par extension, comme une sensation de pression dans la bouche. Le regard, associé aux sensations de pression sur les muqueuses buccales, lors de la tétée, permettrait un étayage sensoriel pour la constitution d'un pare-excitation qui contiendrait et apaiserait les émotions diffuses procurées par les sensations douces.

Dans l'autisme, le démantèlement sensoriel de la zone buccale bloquerait l'intégration des sensations rassurantes de pression sur les muqueuses buccales et pourrait ainsi participer au retrait défensif. La recherche de sensations extrêmes de pression sur les muqueuses buccales par Luc serait alors une tentative pour réunifier et contenir la sensorialité d'une zone buccale «à vif ».

\section{L'APPRÉCIATION DES QUALITÉS SPÉCIFIQUES DES ALIMENTS}

Luc mange sans distinction tout ce qu'il trouve et manifeste peu de capacité de discrimination des goûts ou des qualités des aliments. Ainsi, le plaisir procuré, qu'il soit obtenu par sa langue, le vomi, une brindille de bois ou une purée qu'il fait indistinctement tourner dans sa bouche, semble identique. 
Les qualités gustatives ne sont pas encore reconnues. C'est la sensation tactile sur ses muqueuses qu'il recherche ainsi que des sensations de colmatage d'une zone vécue comme béante. Dans son dossier il est mentionné qu'il s'est un jour intoxiqué en ingérant du ciment. Luc chercherait-il à colmater sa bouche ressentie comme «sans fond »? Les aliments qu'il ingurgite de façon hyperphagique semblent passer à travers lui, ne faisant que traverser son corps, comme s'il n'avait que deux dimensions et qu'il était lui-même une surface trouée.

\section{UN CORPS CREUX SANS DENSITÉ}

Luc est si maigre avec une cage thoracique prédominante, qu'à la piscine, il semble rempli d'air : allongé sur le dos, son corps flotte au point qu'il est pratiquement impossible de le verticaliser. Il remplit d'air sa cage thoracique qu'il bloque ensuite par des apnées. Il faut alors lester ses pieds fortement pour le redresser. Son corps creux rempli de vide, sans poids, semble sans densité.

Luc modifie souvent son rythme respiratoire et peut faire une pause entre ses inspirations et ses expirations, sans se laisser aller à un rythme naturel et régulier. De même qu'il contrôle les entrées et sorties des aliments de sa bouche, il contrôle souvent la sortie de l'air qu'il a inspiré, qu'il l'expire par son nez ou par sa bouche.

Dans son dossier médical, sa maigreur est mentionnée. Il a d'ailleurs été hospitalisé plusieurs fois entre 37 et 39 ans car il criait de plus en plus, se tapait l'abdomen, les cuisses, s'arrachait avec violence la peau et maigrissait. $C^{\prime}$ est à cette période qu'il a été également hospitalisé pour l'intoxication au ciment. Était-ce une tentative de lester son corps pour le rendre plus dense par du poids et une pression interne ou bien une tentative de colmater le vide ressenti au niveau de sa bouche ? Il est à nouveau hospitalisé un an plus tard pour une occlusion intestinale. Ce blocage du transit peut-il être compris comme une rétention qui provoque par dilatation un effet de pression sur les parois intestinales, ce qui permettrait de lutter contre des angoisses de perte de sensations de pression contenantes? Ou bien, est-ce uniquement une lutte contre des angoisses de vidage d'une partie de lui-même au moment de la perte de ses excréments ? La sphinctérisation psychique de ses orifices se constitue difficilement.

Quoi qu'il en soit, malgré le maintien de son appétit, il perd neuf kilos cette année-là et pèse $37 \mathrm{~kg}$. Il gardera un petit gabarit. Même d'un point de vue organique, les aliments ne semblent pas laisser de trace dans son corps. Cela interroge les incidences du fonctionnement psychique sur le fonctionnement métabolique. 
Les travaux de S. Tordjmann 11 montrent combien le fonctionnement de l'organisme du sujet autiste se modifie en fonction de son ouverture psychique sur l'extérieur. Son article qui se réfère à une grille établie en collaboration avec G. Haag évalue des modifications très significatives du système immunitaire selon l'évolution psychique des sujets autistes.

En ce qui concerne Luc, il aurait été intéressant de comparer les variations de la courbe de son poids à son évolution psychique et de repérer l'incidence sur sa prise de poids d'une réintégration de sa zone buccale et d'un corps vécu comme bien fermé par un fond.

\section{L'EMPRISE DES SENS ET LE RECOURS AUX SENSATIONS DOUCES DU STADE FEETAL}

Il existe à la fois chez Luc, un contrôle des sensations qu'il souhaite retrouver ou éviter, et une hypersensibilité aux sensations qu'il perçoit, ce dont il souffre beaucoup. Ceci l'amène à réagir à certains stimuli comme à des excitations insupportables qui seraient pourtant vécues comme anodines chez l'individu normal. Ainsi, au cours de la thérapie, Luc a réagi à la musique par une excitation involontaire et presque douloureuse de sa peau. Il se mettait alors à souffler fortement par le nez tout en frottant l'intérieur de ses mains contre ses cuisses, alternant avec un mâchonnement rapide au moment où il appréciait le plus la mélodie. Le plaisir qu'il s'octroyait était évident tant il se détendait physiquement, balançant un pied en rythme, tout en émettant des « $\mathrm{mmm}$ » de satisfaction. Mais son pare-excitation était si défaillant que l'excitation qui l'envahissait lui provoquait comme un chatouillement irrépressible sur la surface de la peau comme si les vibrations de la musique, sur un mode fœetal, donnaient lieu à des ondes reçues sur toute son enveloppe corporelle.

À force de développer sa sensitivité tactile pour se sentir exister, Luc se voit progressivement envahi par une hypersensibilité et une réactivité nerveuse qu'il ne maîtrise plus. Ce qui est fréquent chez de nombreuses personnes souffrant de troubles autistiques. La vie de Luc semble être sous $l^{\prime}$ « emprise du sensoriel », ce qui bloque ses contenus de pensée en le cantonnant dans des actes de décharge. Luc tente de contrôler ses éprouvés sensoriels qui ne lui laissent que très peu de répit.

Il a développé une technique de modification des éprouvés sensoriels, pour récréer des éprouvés fœtaux, sur le mode de l'hallucinose dont parle

11. S. Tordjmann, Fondation Vallée, Gentilly ; G. Haag, "Grille de repérage clinique des étapes évolutives de l'autisme infantile traité ", dans La psychiatrie de l'enfant, 38, 2, 1995, p. 495-529. 
C. Athanassiou ${ }^{12}$. Ainsi, par les blocages volontaires de sa respiration et par le manque d'investissement de sa densité corporelle à la piscine, il donne l'impression d'être dans un état de flottaison, rappelant l'état d'apesanteur intra-utérin. De même, il s'humidifie le visage à l'écoute de la musique, reconstituant ainsi le vécu des sensations humides du milieu utérin accompagné d'une enveloppe sonore.

Son dossier médical n'apporte aucune précision sur d'éventuels troubles neurologiques à la naissance qui pourraient justifier ses fixations à un stade fœtal.

\section{L'EFFRACTION DU SONORE DANS LA RENCONTRE AVEC L'AUTRE}

La rencontre avec Luc s'est passée dans un premier temps dans la salle commune où je l'ai observé. Je l'ai ensuite suivi en groupe de musicothérapie, puis en individuel. Rencontrer Luc met en difficulté d'utiliser les modalités habituelles de communication du thérapeute : la voix comme le regard. Luc a comme première préoccupation de tenter de se protéger des effractions sonores et particulièrement de celles de la voix.

Dans la salle commune réunissant les quarante-quatre adultes handicapés, Luc manifeste souvent des états de souffrance liés aux bruits et au brouhaha. Il se crispe alors, serre son buste avec un de ses bras, son regard se désorganise et il émet un bruit strident qui « transperce » les oreilles de ceux qui l'entendent, comme pour dire combien il se sent lui-même transpercé par les bruits et le brouhaha, qui le plongent dans une souffrance intolérable. Il peut alors "pleurer", en s'arrachant une touffe de cheveux, comme s'il essayait d'ôter la douleur. Ses réactions aux bruits évoquent des migraines insupportables. Son état de souffrance est visible sur son visage par son expression d'épuisement et des cernes violacées. Pour le calmer, il lui est proposé une promenade à l'extérieur. Il $\mathrm{s}^{\prime} \mathrm{y}$ détend toujours et en revient plus " apaisé » : il cesse de s'arracher les cheveux et relâche globalement sa tension. Ceci rappelle les effets de saturation et de brouillage sensoriel décrits par T. Grandin 13, chez certains autistes qui ont des perceptions indifférenciées sonores, visuelles, tactiles ou gustatives et qui entrent facilement dans une confusion sensorielle intolérable.

Au début de la prise en charge psychothérapique, le fait que je me mette à parler était très problématique. Mes paroles devenaient des bruits insupportables imprévus tout autant que mon regard duquel il se protégeait par

12. C. Athanassiou, Aux sources de la vie psychique : de l'hallucinose à l'hallucination, Lyon, Césura, 1990.

13. T. Grandin, Ma vie d'autiste, Paris, O. Jacob, 1997. 
un strabisme quasi constant. Toute tentative d'entrer en communication avec lui le faisait fuir au fond de la salle, derrière moi, à l'abri de mon regard. Seule la musique rythmée en continu lui était agréable mais sans mes paroles. La continuité mélodique, accompagnée de rythme rappelant les rythmes vitaux cardiaques ou respiratoires, le rassurait. Son regard pouvait se rétablir par instant, ce qui se traduisait par une disparition de son strabisme. Il écoutait la musique, mais dès que je parlais, il déambulait en criant et en tirant les cheveux de son crâne jusqu'à tendre la peau de son visage.

Je me trouvais perçue dans le transfert comme persécutrice dans ses perceptions sonores ou visuelles, tant par mes paroles imprévisibles que par mon regard. Toute tentative de communication l'envahissait et venait effracter son univers en voie de reconstruction. Lorsque je parlais, Luc se raidissait immédiatement et criait en continu en se serrant la cage thoracique. De fait, soit il se détendait et se sentait bien en écoutant la musique, mais je me sentais alors inexistante à force de me taire, soit je parlais et il risquait de se désorganiser encore plus. Quoi qu'il en soit, la perception de la musique accompagnée de ma voix saturait toute possibilité d'intégration sensorielle et entraînait un risque de tomber dans des états de confusion qui se transformaient en douleur sensorielle. En effet, ces états de confusion entraînent une perte de la cohérence interne et de toute capacité d'enchaînement d'un élément à un autre. En recourant au démantèlement et en saturant son canal auditif avec son cri, Luc tente d'apaiser ses ressentis confus et douloureux.

Je mesurais alors quel désarroi pouvaient ressentir des parents d'enfants autistes cherchant juste à exercer leur fonction de parents rassurants. En tant que psychologue, je ne pouvais pas non plus recourir à des paroles habituellement structurantes pour aider à la représentation de vécus archaïques.

T. Grandin ${ }^{14}$, adulte souffrant d'autisme, a décrit la difficulté qu'ont certains autistes à écouter en même temps deux sources sonores différentes. Par exemple un choix s'impose entre écouter une conversation téléphonique ou écouter les bruits de la pièce où se trouve le téléphone. Leur difficulté peut aller jusqu'à ne plus distinguer quoi que ce soit, dans une confusion sensorielle. De même il est impossible pour d'autres personnes autistes d'investir deux canaux sensoriels différents, par exemple de s'apercevoir qu'un chat est monté sur les genoux alors que l'attention est focalisée entièrement sur l'écoute du téléphone.

Le pare-excitation de Luc était si défaillant qu'il se coupait des échanges avec autrui pour se protéger. Deux données sensorielles n'étaient pas inté-

14. T. Grandin, Penser en images et autres témoignages sur l'autisme, préf. De O. Sachs, Paris, Odile Jacob, 1997, p. 69-91. 
grables pour lui tant qu'elles n'étaient pas encore différenciables. Ainsi, lorsque j'acceptais de "disparaître » de son champ sonore, j'observais chez Luc une détente et un réel plaisir à écouter la musique en ma présence. Il se détendait surtout avec de la musique classique. Il détestait les chansons à deux voix. Il lui arrivait de mettre sa tête près du poste de radio en balançant en rythme un de ses pieds. Il lui arrivait également de plaquer son buste contre la peau des congas, instruments de musique avec lesquels je jouais parfois.

\section{LE TEMPS DU FILTRAGE SENSORIEL}

Il m'a donc fallu sélectionner dans un premier temps les stimuli auditifs que je lui proposais, en envisageant un par un les stimuli sensoriels que j'émettais. Pendant plus d'un an, j'ai appris à reconnaître ce qui gênait ou favorisait sa détente. Mes repères de techniques en ont été bousculés, je suis passée par un moment de déprime intense. Je renonçais à apporter un soulagement à Luc, par tout ce qui pouvait émaner de ma personne, comme les mots et même ma voix, hors mis ma présence silencieuse. Je me suis retrouvée contrainte à bouger le moins possible dans la pièce, à ne presque plus parler, ni le regarder quand il se mettait derrière moi dans un évitement du regard, et à juste écouter nos présences enveloppées par la musique. À chaque changement de musique, il se raidissait pendant plusieurs minutes, le temps nécessaire pour capter la régularité rythmique du nouveau morceau jusqu'à ce qu'il se détende.

En me faisant éprouver ce renoncement à moi-même, je pense que Luc me faisait partager dans le transfert son propre renoncement à lui-même dans la relation et son désespoir intense quant à ses propres capacités à entrer en contact avec autrui. Son sentiment d'exister se fragilisait à chaque changement impromptu. Seule l'écoute de la musique en continu dans la présence de l'autre pouvait l'apaiser. Ce n'est qu'à partir du moment où j'ai accepté que notre simple présence sans autre manifestation soit enfin adéquate pour construire un pare-excitation à ses difficultés et angoisses, que Luc a commencé à manifester de plus en plus de comportements nouveaux. La simple présence attentive dans un cadre transférentiel construisait un pare-excitation à ses ressentis trop intenses et favorisait un remantèlement en reliant ses ressentis.

Mon premier rôle, dans le transfert a été celui de filtre, étayé par l'écoute contenante de la musique. J'ai joué ce rôle sans vraiment en mesurer l'ampleur au début, car je me suis laissée penser dans des moments de doute que Luc se détendrait aussi bien sans ma présence. C'est à ce moment qu'il a 
manifesté qu'il attendait sa séance et que l'écoute musicale l'intéressait peu si je n'étais pas là.

\section{LE TEMPS DES ÉCHANGES AQUEUX}

Dans un deuxième temps, je suis devenue dans le transfert, une sorte d'enveloppe humide, sans doute celle-là même qu'il appliquait sur son visage après avoir léché sa main. Si Luc protégeait son nez et sa bouche en les cachant avec sa main, au début de nos rencontres, il avait fini par régulièrement humidifier son visage de sa salive en se frottant largement toute la face, comme pour accompagner un réveil sensoriel du visage. Or, un jour où la thérapie semblait très peu avancer, Luc a déposé très savamment un filet de salive sur mon écharpe que j'avais déposée sur une table. Cela m'a beaucoup émue tant je savais combien il contrôlait ce qui sortait de sa bouche. J'ai eu la confirmation de cette manifestation transférentielle lorsqu'il a déposé d'une façon assez extraordinaire un filet de bave à une autre reprise, sur la tranche d'une de mes clefs, filet qui est resté suspendu sur «ce rebord ». La rencontre était établie par ses substances : je devais en effet reprendre cette clef en fin de séance.

J'avais alors l'impression d'une incitation à un contact extrêmement intime avec cette partie archaïque en lui. D'une certaine façon, il me faisait entrer dans l'univers de sa cavité buccale en déposant sur moi une substance corporelle. Je devenais sa cavité buccale pour contenir sa substance. J'étais mise en équation adhésive avec une partie de lui-même en tant qu'enveloppe humide.

Par moment, Luc commençait à éprouver plus nettement du plaisir à l'écoute musicale, et se protégeait moins de ma présence, ce qui engendrait une excitation importante. Cela l'amenait à se frotter la peau tout en soufflant intensément. Il émettait alors de nouveaux sons et des mimiques, qui manifestaient un mélange de plaisir, de rire et de ricanement, comme si la circulation sanguine en surface revenait et le mettait dans des ressentis de picotements ou de chatouilles qui devenaient gênants.

À d'autres moments de larges sourires éclairaient son visage et il « mâchonnait » la musique tout en émettant des « mmm » de satisfaction, les yeux plissés, serrant ses épaules pour mieux ressentir son dos, comme il pouvait le faire quand il savourait la nourriture qu'il avait volée. Il aimait la musique comme il aimait la nourriture, et tout cela semblait lui procurer également des sensations gustatives et des sensations contenantes au niveau de son dos. En dehors des séances, il se montrait moins vulnérable aux bruits extérieurs, ce qui témoignait de l'installation d'un pare-excitation qui commençait à filtrer l'insupportable. 


\section{L'INCORPORATION DE LA FONCTION DE FILTRE PARE-EXCITANT}

Le troisième temps de la thérapie a permis à Luc d'installer le regard et la possibilité d'une différenciation entre plusieurs sens dont il pouvait désormais accepter la coexistence de perception sans ressentir d'impression de brouillage sensoriel. Cette phase a débuté par un mouvement d'incorporation du thérapeute.

En effet, un jour, en séance, alors qu'il éprouvait du plaisir à l'écoute de la musique et qu'il balançait son pied en rythme, Luc s'est arrêté, m'a lancé un regard fortement pénétrant, comme s'il s'imprégnait de moi, et s'est vite retourné en mâchonnant vivement comme il le faisait à la dérobée après avoir volé de la nourriture. Je me suis sentie alors contre-transférentiellement volée, mangée, « dévorée des yeux » dans le sens littéral. J'avais l'impression d'avoir été " saisie par une main » avec la rapidité d'un éclair et mise en bouche tant l'ensemble des gestes qu'il faisait était identique lorsqu'il volait de la nourriture à la table voisine. Il se dépêchait alors de mâchonner, l'esprit en alerte et le corps replié, dans la crainte qu' on lui reprenne ce précieux bien.

L'assimilation qu'il fait entre regarder et dévorer, m'a fait immédiatement comprendre l'intensité de sa peur d'être dévoré et mâchonné lorsque je le regardais. À partir de cet éprouvé d'angoisse revécu dans le transfert, il a pu associer du visuel au plaisir de mâchonner et au plaisir de l'écoute musicale. Cet échange de regard pendant le plaisir oral se rapproche d'une situation habituelle de nourrissage mère-enfant lors de laquelle le plaisir tactile et gustatif de la tétée s'associe aux sensations dorsales de soutien et à l'échange de regard. J'assistais à l'émergence, chez Luc, d'une consensualité qui s'instaurait dans la relation transférentielle. C'est-à-dire qu'il pouvait désormais associer différents sens, sans confusion. Dès lors pour lui, le regard semblait perdre sa dimension persécutrice de dévorer ou se sentir dévoré, de piquer ou se sentir piqué ou bien encore de se sentir immobilisé. Je parle de sensations de piqûres car il existe à la surface de l'œil des cellules qui provoquent des sensations de piqûres lorsqu'elles sont excitées par la lumière. Chez Luc, l'intensité du regard s'étant apaisée grâce au " remantèlement ", il n'était plus dans des vécus de saturation sensorielle.

À partir de ce moment-là, il se met à me regarder de plus en plus facilement, par exemple à mon arrivée dans la salle commune. De loin, il me regarde désormais droit dans les yeux et sans aucun strabisme. À distance, je peux maintenant lui adresser des paroles, mettre des mots en ayant l'impression qu'il les accepte alors que cela le dérangeait auparavant. J'ai l'impression qu'enfin le regard n'est plus une perception isolée aux effets inquiétants mais devient une " partie d'un tout " c'est-à-dire métonymique 
d'un échange, d'un soutien humain de qualité. Ce qui traduit sa sortie d'un autisme profond par la constitution d'un pare-excitant défaillant jusqu'alors.

Un travail sur l'émergence de représentations de ses états mentaux reste à mettre en place. Une certaine cohérence de ses perceptions désormais différenciées s'est installée. La trace d'éprouvés corporels de mieux-être au contact du thérapeute a fait office de bon contenant et a relancé l'organisation de l'oralité. Si cette trace psychocorporelle a commencé à construire un moi corporel chez Luc, elle reste cependant encore très peu psychisée.

Il apparaît à travers ce suivi, que la réversibilité du démantèlement est toujours possible même à l'âge adulte. Dans ce cas clinique, il a fallu quatre ans de suivi thérapeutique avant de voir émerger un accès à la consensualité. Dans un premier temps, le thérapeute a eu un rôle de pare-excitation et de filtre sensoriel de façon très concrète. Puis il semble avoir été investi dans le transfert comme un contenant humide à l'image d'une enveloppe utérine, ceci se manifestant par l'intermédiaire de ses substances corporelles. C'est dans un troisième temps que sont apparues les tentatives d'incorporation du thérapeute, devenu alors " pôle d'attraction » et de focalisation des différentes modalités sensorielles. En effet, le plaisir de l'écoute s'est alors associé au plaisir d'attraper par le regard et de "déguster».

Le rôle de pare-excitant du thérapeute a permis à Luc de trouver ses propres stratégies pour faire face à l'excitation. La réduction sélective des stimuli auditifs, en particulier ceux de la voix, maintenus suffisamment longtemps pour qu'il en fasse l'expérience de s'en trouver apaisé, a étayé la possibilité pour lui de faire « un tri » dans la quantité d'excitation qu'il recevait. Un comportement de décharge de l'excès d'excitation s'est mis en place, remplaçant l'ancien recours au démantèlement.

Une fois le " pare-excitation » constitué, le dedans s'est différencié du dehors. Un temps de plaisir retrouvé à chaque séance devenait possible et facilitait la constitution d'un bon objet.

Par ailleurs, l'ensemble des éléments cliniques présentés souligne l'importance de la recherche de sensations de pression sur les muqueuses buccales et sur tout le corps, associée aux sensations humides comme pour recréer les sensations intra utero.

Les sensations de pression sur le corps fréquemment recherchées par cet homme, particulièrement sur l'intérieur de sa cavité buccale, montrent combien l'excitation agréable de cette zone peut remonter à l'étape fœtale, le liquide amniotique provoquant déjà des sensations de pression sur les muqueuses comme sur toute l'enveloppe corporelle. Ceci amène à concevoir 
un temps pré-natal au stade oral tel qu'il est décrit par Freud 15, qui préparerait le lien ultérieur entre le besoin alimentaire et le désir de satisfaction. Il y a un en de-çà de la rencontre sein (ou biberon) et bouche. Depuis les échographies, nous savons que la plupart des fœtus sucent leur pouce in utero, et que plus rarement, certains avalent du liquide amniotique. Il apparaît aux vues de toutes ces données que chez le fœetus, la sensibilité et l'activité de la zone orale sont déjà développées.

L'excitation des muqueuses internes de la bouche par un liquide est un phénomène " ressource " ou "transitionnel ", contenant, un des premiers modes de maintien d'une sensation d'exister pour le bébé. À la naissance, il existe une impression de se perdre dans l'espace, de perdre la pression aqueuse de la «masse » enveloppante placentaire. Il en va de même pour la bouche qui pourrait ainsi ne plus être ressentie, lorsque seules les perceptions de la couche externe de la peau du visage ou des muqueuses buccales seraient investies sans aucune pression tant que la contenance relationnelle n'a pas encore pris le relais.

Face à cette déferlante d'angoisse d'annihilation dans une conscience trop précoce de la séparation mère-bébé, l'enfant en serait réduit à recréer de façon régressive des sensations fœtales en recourant à ses propres liquides et substances internes, dans des recherches de sensations d'un environnement humide et par la recherche de sensations de pression pare-excitante et contenante. Cette perspective éclaire ce que Francès Tustin appelle « la prise de conscience trop précoce de la séparation ».

Quoi qu'il en soit, ce travail thérapeutique bouscule nos représentations normalement basées sur l'intégration et l'association de données sensorielles différenciées avec un pare-excitation suffisamment constitué. Le suivi de cet adulte autiste nous entraîne à élargir nos représentations de l'investissement des zones corporelles, particulièrement celui de la cavité buccale.

Les hypothèses avancées à partir du cas de Luc restent à être confrontées avec d'autres cas cliniques d'adultes souffrant d'autisme.

\section{BIBLIOGRAPHIE}

AnZIEU, D. 1985. Le moi-peau, Paris, Dunod, 254 p.

Athanassiou, C. 1990. Aux sources de la vie psychique : de l'hallucinose à l'hallucination, Lyon, Césura.

BABABE, N. 1999. La sensorialité au regard du modèle de l'autisme, thèse de doctorat de l'Université Paris VII, sous la direction de P. Fédida.

15. S. Freud, "La sexualité infantile », chapitre II, dans Trois essais sur la théorie de la sexualité, Paris, Gallimard, 1962. 
BICK, E. 1968. "The experience of the skin un early object-relations », int. Journal of Psychoanalyse, 49, 484-6, trad. franç. G. et M. Haag, "L'expérience de la peau dans les relations d'objet précoces », dans Meltzer D., et coll., Explorations dans le monde de l'autisme, 1975, p. 240-244.

FEDIDA, P. 1971. «L'anatomie dans la psychanalyse », dans Nouvelle revue de psychanalyse, $\mathrm{n}^{\circ}$ 3, p. 109-126.

FREUD, S. 1962. «La sexualité infantile », chapitre II, dans Trois essais sur la théorie de la sexualité, Paris, Gallimard.

Grandin, T. 1994. Ma vie d'autiste, Paris, Odile Jacob, sept.

GRANDIN, T. 1997. Penser en images et autres témoignages sur l'autisme, Paris, Odile Jacob, $261 \mathrm{p}$.

HAAG, G. 1995. « Grille de repérage clinique des étapes évolutives de l'autisme infantile traité », dans La psychiatrie de l'enfant, 38, 2, p. 495-529.

LHEUREuX-DAVIDSE, C. 2003. L'autisme infantile ou le bruit de la rencontre, Paris, L'Harmattan.

Le Poulichet, S. 1994. «La boulimie et le temps cannibalique », dans L'œuvre du temps en psychanalyse, Paris, Rivages, Psychanalyse, p. 181-203.

MAIELLO, S., 1991. «L'Oracolo. Un esplorazione alle radici della memoria auditiva », dans Analysis. Rivista Internazionale di psicoterapia clinica, Anno 2 n 3, p. 245-268, trad. franç., « L'objet sonore. L'origine prénatale de la mémoire auditive ; une hypothèse ", dans Journal de la psychanalyse de l'enfant, $\mathrm{n}^{\circ} 20$.

Meltzer, D. 1975. Explorations in Autism, Roland Harris Trust, Clunie Press, trad. franç., Explorations dans le monde de l'autisme, Paris, Payot, 1980, 259 p.

PIERON, H. 1974. La sensation, coll. Que sais-je ?, n 555, Paris, PUF.

ToRJMAnN, S. Fondation Vallée, Gentilly.

TUSTIN, F. 1990. The protective shell in children and adults, London, Karnac books, trad. franc., A.L. Hacker, Autisme et protection, Paris, Le Seuil, 1992.

Tustin, F. 1989. Autistic Barriers in Neurotic Patients, Londres, Karnac Books, trad. franc., Le trou noir de la psyché, Paris, Le Seuil, oct. 256 p.

WinNicotT, D. 1975. « La crainte de l'effondrement », dans Nouvelle revue de psychanalyse, 11, 35-44.

\section{Résumé}

Le suivi psychothérapeutique d'un adulte autiste de 44 ans, nous conduit à observer les effets du démantèlement à long terme sur l'investissement de sa zone buccale et sur l'abord de son environnement. La zone orale non érotisée apparaît comme réduite à un ensemble d'éléments vécus comme disparates : la langue, les muqueuses, la salive, etc. Chacun étant susceptible de procurer des effets sensoriels précis assurant une fonction rassurante ponctuelle. Cette prise en charge thérapeutique a nécessité un aménagement du cadre. La psychothérapeute a tenu, dans un premier temps deux fonctions particulières : celle de pare-excitation et celle d'enveloppe humide. Ensuite elle a pu être " intégrée » comme soutien et pôle d'unification des sensations démantelées. De là, une consensualité a pu émerger chez ce patient. L'ampleur des moments de recherche de sensations de pression et de sensations humides manifestée par cet 
adulte autiste, nous a conduit à postuler l'hypothèse de l'effet contenant des sensations de pression sur les sensations plus douces de surface, ceci en lien avec les vécus intra-utéro. Ces données restent à être confrontées à d'autres cas d'adultes autistes.

Mots clés

Autisme, démantèlement, confusion sensorielle, oralité, fonctions de la langue, sensations de pression, sensations feetales, contre-transfert.

SENSORIAL DISMANTLEMENT REVERSIBILITY IN AN AUTISTIC ADULT

Summary

The follow up of an autistic adult psychotherapy allowed us to observe the long-term effects of the sensorial dismantlement on the buccal area and on the perception of his environment. The non-erotised buccal area seems reduced to a group of elements perceived as disparate : the tongue, the mucous membranes, the saliva, etc... Each element is able to provide precise sensorial effects assuring a punctual reassuring function. The therapy requested some adjustments. At first the therapeutist had two particular functions : she was an excitement shield and a humid blanket. Then, she was able to be integrated as a support and a unification pole of the dismantled sensations. Later, a harmony between the sensations was able to emerge from the patient. The length of the times spent by the patient to seek pressure or humid sensations led us to emit the hypothesis that the pressure sensations had a moderating effect on the softer superficial sensations which is linked to the intra-utero experiences. This data needs to be compared with the results of other studies of autistic adults.

Keywords

Autism, dismantlement, sensorial confusion, orality, tongue functions, pressure sensations, fotal sensations, counter-transference. 